\title{
The challenges of using feedback from patients to inform design
}

\section{EDITORIAL}

\author{
Bev Fitzsimons
}

The Point of Care Foundation, London, UK

\author{
To Cite: Fitzsimons B. The challenges of using \\ feedback from patients to inform design. JHD. \\ 2018;3(2):124-126. \\ https://doi.org/10.21853/JHD.2018.59

\section{Corresponding Author:} \\ Bev Fitzsimons \\ The Point of Care Foundation \\ London, UK \\ BevFitzsimons@pointofcarefoundation.org.uk

\section{Copyright:} \\ (C) 2018 The Authors. Published by Archetype Health \\ Pty Ltd.. This is an open access article under the CC \\ BY-NC-ND 4.0 license.
}

\section{SUMMARY}

A range of sources of data on patients' experiences exist. There is greater emphasis on using quantitative data for the purposes of assurance and accountability than there is on using the range of data for improvement. Patients' legitimacy in commenting on aspects such as communication and coordination of care is accepted. Less so, the potential for patients to contribute to consideration of safety issues. A culture of curiosity is a prerequisite for making best use of patient insight data for improvement. There is also considerable appetite and potential for greater involvement of patients in the co-design of improvements in care.

\section{Key Words}

Patient experience; patient feedback; patient involvement; experience-based codesign; design thinking

\section{INTRODUCTION}

This issue of The Journal of Health Design shares some of the innovative approaches to collecting feedback from patients that are used to inform the design of health services. While a range of approaches is possible, the selection of which is most appropriate is fraught with complexity for many healthcare providers.

The nub of the issue is the purpose for which feedback is sought: the concept of healthcare design can mean different things to different people. It can mean re-design of services as a result of summative service evaluation, or it can mean incremental improvements in services as a result of ongoing formative evaluation.

Two further complicating factors are the range of alternative sources of patient experience data from within the system that can be used for service design, and the domains for which patient feedback is sought, in order to aid design.

Healthcare providers have multiple sources of data at their disposal: in the United Kingdom (UK) this includes regulatory reports, professional inspection reports, clinical audits, analyses of routinely collected data from numerous bodies, as well as national and local collections of data from patients and families. There is a national survey programme and the "family and friends test", to name but two, alongside local improvement projects using innovative methods such as The Point of Care Foundation's Sweeney programme, which uses methods such as Experience-Based Co-design, and Patient and Family Centred Care.

Many people whose roles include the collection and analysis of patients' experience data report the complexity of making sense of the range of data coming as it does in different formats, covering different timescales, and of varying quality. "Patient experience teams are sometimes so busy gathering data and compiling reports, that less time is available to do something with the data. Efforts to improve services are in danger of being squeezed out."

Compounding this is a justifiable resistance from patient activists, feeling that they provide the system with "feedback fodder" without meaningful involvement, sharing of power and engagement in the service response, and the appetite for true partnership in co-designed responses to patient (and other) feedback. ${ }^{2}$

Commonly, patients are asked to comment on what could be defined as "patient-centred" aspects of care. Using the Institute of Medicine definition, this includes dimensions such as: whether care respected patients' values, preferences, and expressed needs; the coordination and integration of care; the provision of information to patients; their physical comfort; emotional support and alleviation of anxiety; the involvement of family and friends; continuity of care; and access to care. ${ }^{3}$ 
Whilst these dimensions are important, it is interesting that this realm of "patients' experience" on which patients should have a legitimate expectation to express their views is often considered as a separate dimension of healthcare quality than that of safety and clinical effectiveness. Yet it is arguable whether these are indeed separate dimensions, or whether experience overarches them all. ${ }^{4}$

In terms of effectiveness, there is growing acceptance that the outcomes that are defined and evaluated by the patient such as Patient Reported Outcome Measures (PROMS), and Patient Reported Experience Measures (PREMS), represent the value of health care to patients and should be included alongside clinical assessments of the effectiveness of care. ${ }^{5}$ Less readily accepted is that patients can also provide a valuable voice in matters of safety. ${ }^{6}$

Yet patients' accounts may provide a "smoke detector" of problems in the system, rather than an "alarm" when something has already gone wrong. The problem may be one of epistemic injustice, in which the testimony of those who are considered to have "expert knowledge" is privileged above patients, whose testimony is not considered expert in the technical sense. ${ }^{7}$

Regardless of the approach taken to solicit feedback from patients to inform service design, it is clear that it is not easy for professionals to act on such information. Sheard et al. identify some factors that make it more likely that feedback from patients is successfully acted upon. These include that:

- data are believed

- staff must believe that listening to patients is a worthwhile exercise from which they can gain valuable insight

- staff are empowered to act on the data

- a higher level of organisation commitment to acting on the data may be needed. ${ }^{8}$

The organisations that are most successful at not only collecting data but using it for improvement, approach the data with a curiosity about what could improve. This is what Dixon-Woods described as "problem-sensing" behaviour, which results in active seeking out of weaknesses and devising responses to strengthen their organisations. In contrast, other, less positive, behaviours are described as "comfort-seeking", in which the focus is much more on external impression management and seeking reassurance that all was well, thereby creating less insight. ${ }^{9}$

As well as the factors that Sheard identifies as critical to making the best use of patient experience data, we at The Point of Care Foundation would add an additional dimension: that patients are meaningfully involved in the design of services, as well in providing data to inform them in an effort to create truly patient-centred care.

\section{REFERENCES}

1. Sibley M. Understanding patient experience is fundamental to a patient centred service vision. 24 April 2018. [Accessed 2018 MAY 1]. Available from: https://blogs.bmj.com/bmj/2018/04/24/miles-sibley-understandingpatient-experience-is-fundamental-to-a-patient-centred-service-vision/

2. Gilbert D. Why we need patient leaders. 21 September 2017. [Accessed 2018 May 1]. Available from: https://blogs.bmj.com/bmj/2017/09/21/david-gilbert-why-we-need-patient-leaders/

3. Institute of Medicine. Crossing the quality chasm: a new health system for the $21^{\text {st }}$ century. Washington DC: National Academy Press; 2001.

4. Wolfe JA. The consumer has spoken: patient experience is now healthcare's core differentiator. Patient Experience Journal: Vol 5; Iss 1; Article 1. Available from: http://pxjournal.org/journal/vol5/iss1/1

5. Black N, Varaganum M, Hutchings A. Relationship between patient reported experience (PREMs) and patient reported outcomes (PROMS) in elective surgery. BMJ Qual Saf. 2014 Jul;23(7):534-42. doi: 10.1136/bmjqs2013-002707

6. O'Hara JK, Reynolds C, Moore S, et al. What can patients tell us about the quality and safety of hospital care? Findings from a UK multicenter survey study BMJ Qual Saf. 2017;0:1-10. doi:10.1136/bmjqs-2017-006974

7. Fitzsimons B, Cornwell J. What can we learn from patients' perspectives on the quality and safety of hospital care? Editorial. BMJ Qual Saf Published Online First. 05 April 2018. doi: 10.1136/bmjqs-2018-008106 


\section{The
Journal of
Health Design}

8. Sheard L, Marsh C, O'Hara J, et al. The Patient Feedback Response Framework-Understanding why UK hospital staff find it difficult to make improvements based on patient feedback: A qualitative study. Soc Sci Med. 2017;178:19-27.doi:10.1016/j.socscimed.2017.02.005

9. Dixon-Woods M, Baker R, Charles K, et al. Culture and behaviour in the English National Health Service: overview of lessons from a large multimethod study. BMJ Qual Saf 2014;23:106-15.

\section{ACKNOWLEDGEMENTS}

None

\section{PEER REVIEW}

Not commissioned. Externally peer reviewed.

\section{CONFLICTS OF INTEREST}

The authors declare that they have no competing interests.

\section{FUNDING}

None

ETHICS COMMITTEE APPROVAL

None 